\title{
Localized Motion Abetment Sensor Deployment Method for Hole Recognition and Curative
}

\author{
Pradnya Irawadkar
}

\author{
Archana Lomte
}

\begin{abstract}
A basic service in Wireless sensor network is monitoring of particular region of interest since due to the nature of WSN random development hole in the ROI is obvious. So for these reason ROI should be completely and continuously covered. And in this paper we have attended and covered the issue of hole detection and healing in mobile WSNs. The main drawbacks of existing systems are selecting the boundary of ROI, finding coverage holes and estimating their characteristics, finding best location for relocating mobile nodes for repairing holes, and moving mobile nodes to safe location with minimum moving and messaging cost

And to handle all these problems a novel technique is present named hole detection and healing. The computational complexity of HEAL is given as (v2), in which is the average number of 1-hop neighbors. In Heal there are Two Stages first, it detects boundary nodes and finds holes and in second, it treats hole healing. A distributed virtual forces-based local healing methodology is presented in which the nodes in a suitable distance from the hole will be included in the healing procedure and also presented wakeup scheduling algorithm. with the help of wakeup scheduling algorithm energy utilization of sensor nodes is reduced and increases the life of network.
\end{abstract}

\section{Keywords}

Hole detection and healing, Border nodes, Region of Interest, Wireless sensor network, Wake up Scheduling.

\section{INTRODUCTION}

From last few years there has been lot of research done in Wireless Sensor Network (WSN). A Wireless Sensor Network (WSN) consists of sensors with restricted computational and communication power. For the number of different failures Sensors are very fragile and suspicious, for example, sudden shock by their deployment or depletion of their restricted energy assets. for these reason fragility must be viewed as a typical property of the network. Number of anomalies can happen in WSNs due to which their functionalities causing various types of holes, to be specific: routing holes, coverage holes, jamming holes and worm holes, are weaken [13]. In this paper bounded holes which are very large are covered, that is, large holes that are described by sensor nodes. For this situation, coverage holes which mean ranges not secured by any node and communication holes which mean regions without any nodes.

One of the important services provided by a WSN is the monitoring of a determined region of interest (ROI), where it senses the fundamental obligation in the environment and covey's the data to the sink. Which Guarantee's that the ROI is totally secured at all time is important [6].

Never the less, we cannot avoid the emergence of holes in the ROI because of the nature of WSNs, random deployment, environmental variables, and external attacks. Hence, the event which happens inside these holes is not discovered or reported and, so the main task of the network will not be finished. Accordingly, it is of prime Importance to give a selforganizing system to detect and recoup holes. The whole detection and healing issues is addressed in this paper. Most of techniques which are existing use global operation for calculating the size of big hole and place a group of mobile nodes to heal the hole. Some current techniques need solid forwardness and even impractical ones.

Hole Detection and Healing (HEAL) is the solution presented in this paper, to avoid these drawbacks of existing system, also has low complexity. HEAL algorithm is a distributed and localized that works in two unique stages. The first stage is divided in three subtasks; hole identification hole discovery (HD) and border detection. Not like previous approaches, a distributed and localized hole detection algorithm (DHD) that works as a replacement over the Gabriel Graph (GG) of the network. DHD has a low complexity and manages holes of different structures and sizes regardless of the nodes distribution and density. The second stage treats the hole healing with hole healing area (HHA). It comprises of two sub-errands; hole healing area determination and relocation of node. Also it present's wakeup scheduling algorithm. By using wakeup scheduling algorithm, energy utilization of sensor nodes is reduced and due to which increases the life of network.

\section{RELATED WORK}

This section is parted in two different areas, which are:
A) Hole and Border Detection.
B) Coverage Enhancement and Hole Healing.

\section{A. Hole and Border Detection}

Lot of research has been done in the hole and border detection problem [9] [10] [11] [14] [15] [16]. In the study by F. Stefan [14], they have stated a simple algorithm for identifying holes in a wireless communication network that is depended on the connectivity of the communication chart. This has applications in the interpretation and assessment of information gained through a wireless sensor network, since big holes in the communication chart are caused by events that the sensor network was installed to screen in any case. They have portrayed further applications of their hole finding routine, where the knowledge of holes in the network accommodates better performance of existing topology-based, location-free conventions. While with the help of their experiments they suggested that the introduction of hole detection methodology is suitable just in the setting of large, rather dense communication graphs, late deployments of wireless sensor networks have reached at such densities and sizes of a number of thousand nodes.

N. Lehmannet al [15] explained that constrained stress centrality is a helpful index for extracting topological boundary data from a geometric sensor network, provided that 
the distribution of node takes after an appropriate random distribution. As this is solid presumption, it seems desirable to think of more general techniques. Moreover, a methodology based on random distributions may even now fail in some uncommon cases despite the fact that the probability of failures is very low, so it is especially interesting to develop deterministic techniques for boundary recognition.

The new boundary detection algorithm proposed by Y. Wang et al [10] proved that discovers boundaries in the continuous case, in discrete sensors networks a number of implementation issues emerge. Initially, actually for a given homogony type; there need not be a unique shortest path Among two nodes .Subsequently, the boundary cycle found by their algorithm, as explained in the simulations, may not firmly encompass the real boundaries. They have given two methodologies to enhance it. First is to use the nodes with lower degree can be on the boundary; so that they developed a preferential plan for low-degree nodes when calculating shortest paths. Second methodology is to utilize a repetitive technique to discover more external nodes, and afterward refine the boundary; this can nearly help to attain the problem that few external points may have the same positions as they utilize hop counts to approximate true distances. Secondly, choosing the right orderings of the external nodes requires some care. In the continuous case, external nodes project to their nearest node in the inner boundary that gives a slow and continuous ordering of external nodes. In the discrete case, since they utilize hop count to appropriate the true distance, it is conceivable that different external points are mapped to the same position on the inner boundary, clouding their ordering. Q. Fang et al [11] Explained that holes are critical topological features that need to be studied.

In routing, gaps are communication voids that cause greedy sending to fail. Gaps Holes can likely characterize to signify regions of interest, for example, the"hot spot" built by traffic congestion. They defined holes to be the region enclosed by a polygonal cycle which consist all the nodes where nearby minima can be found. They proposed simple and distributed algorithms, the Tent rule and Bound Hole, to recognize and assemble routes around holes. They demonstrated that to check the boundaries of holes Bound hole is used.

\section{B. Coverage Enhancement and Hole Healing}

In a mobile WSN, one of the goals of the development is to increase area coverage. A number of methods for enhancing network coverage exist in the literature. Algorithms that relate this class consists [2] [3] [4] [5] [12] [17] [18], to name some.

In coverage pattern-based movement [8], [7], the target areas for mobile nodes are calculated based on a predefined coverage Pattern, while comparison is made between mobile nodes and electromagnetic particles in virtual forces-based movement [8] [7]. In the study by Wang et al. [12], they proposed three distinctive deployment protocols to relocate mobile sensors once coverage holes are detected by using Voronoi diagrams. S. Ganeriwal et al [17] proposed a plan called Co-Fi that relocates mobile nodes to replace low energy nodes.

C.Y. Chang et al [3] presented three hole-movement methodologies for moving a current huge hole in such a manner that either the total energy consumption is minimized or the power consumption of sensors is balanced. In a hybrid network comprising of both stationary and mobile sensor nodes, one goal for utilizing mobile sensor nodes is to reduce coverage holes made by those stationary nodes [2] [18]. C.Y. Chang et al [2] solve the coverage issue by making use of a moving robot. They presented a tracking technique and a robot repairing algorithm. The tracking technique leaves the robot's footmark on sensors to learn better routes for sending repairing requests to the robot while the repairing algorithm builds an effective path that passes through all failure areas. Li et al. [4] present a group of localized robot-assisted sensor relocation algorithms, by which mobile robots pickup inactive sensors and convey them to encountered sensing holes. Nadeem et al. [5] presented MAPC, a mobility assisted probabilistic coverage for improving and keeping up the region coverage by moving mobile sensor nodes to strategic positions in the uncovered range.

\section{PROPOSED SYSTEM}

To avoid the drawback of existing system, a technique is explained here; called holes detection and healing (HEAL). There are two stages in HEAL.

A) In the first stage, there are three sub tasks those are hole identification, hole discovery (HD) and border detection. Also distributed and localized hole detection algorithm (DHD) is presented which operates on Gabriel graph (GG) of the network.

B) In second stage, there are two sub tasks those are hole healing area determination and node relocation. Additionally wakeup scheduling algorithm is presented. After healing process sensor nodes Acts as dead when they do not want to send data. If any node wants to send data then it finds the shortest path source to destination and wakeup those sensor nodes in the path for sending data using wakeup scheduling algorithm. Due to wake up scheduling we can reduce energy utilization of sensor nodes and increase the life of network.

\section{A.Algorithm 1}

Wake up Scheduling Algorithm:-

1: Perform logical space coordinate space construction using the AODV protocol.

2: Let $\mathrm{K}$ be the time for which we wish to run the protocol.

3: Let $n 1 s, n 2 s, n 3 s, n m s$ be the total number of m nodes, who select sleep mode as they don't have to send data.

4: Let nsrc be the source node who wants to send the data to destination. It obtains the path to the destination node $\mathrm{S}$ using the AODV protocol.

5: nsrc then creates the list of nodes in the shortest path to destination.

6: nsrc then generates the wake-up request for these sleeping nodes.

7: After a response from all sleeping node, nsrc starts data transfer.

\section{B. Mathematical Model}

1) Hole Detection: Distance (bm , bn) $=$ Max \{ Distance $(b j$, bk)/bj, bk_b0; b1, . , bNg .

Then, it calculates the hole center, which is the mid-point $\mathrm{v}$ of segment bm_bn

$\mathrm{xv}=(\mathrm{xbn})=2$

$\mathrm{yv}=(\mathrm{ybn})=2$

Where,

bi is node.

$\mathrm{fb} 0 ; \mathrm{b} 1 ; \ldots ; \mathrm{bNg}$ are Boundary Nodes 


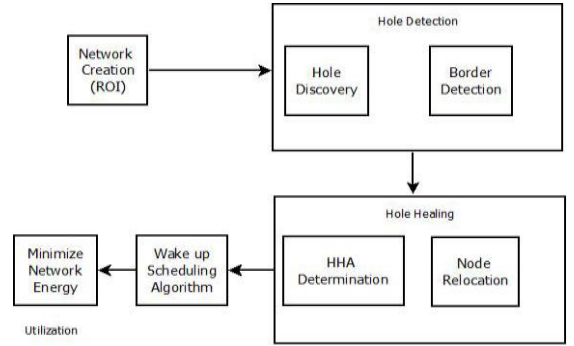

Fig.1: System Architecture

2) Hole Healing: $R=r_{-}\left(1+{ }_{-}\right)$, _ $R+$

Where $r$ is the hole radius._ is a positive constant, which depends on the nodes density and the sensing range Rs.

3) Shortest Path Calculation: $\mathrm{p}(\mathrm{x} 2 \square \mathrm{x} 1) 2+(\mathrm{y} 2 \square \mathrm{y} 1) 2$ Where, $x 1 ; x 2, y 1 ; y 2$ are co-ordinates of sensor node

4) Energy Calculation: ETx data $=$ Eelec_k-bit $/$ message + eamp_k_d ${ }^{\wedge} 2$

Where, Eelec is energy required to send data. Eamp is ampere constant. $\mathrm{d}$ is distance between sensor nodes

\section{RESULTS AND DISCUSSION}

The system is built using Java (Version JDK 8) to evaluate the efficiency, effectiveness. The development tool used is NetBeans (Version 8). The experiments performed on Core2Duo Intel processor, 2GB RAM under Windows XP Professional. The system does not require any specific hardware to run; any Standard machine is capable of running the application table 1 shows approximate analysis of existing system and proposed system with respect to network size and average energy (Joules) of nodes. Energy of nodes is depleted due to computational overhead, so network life is decreased.

Table 1. Comparison of proposed work with respect to energy consumption

\begin{tabular}{|c|c|c|}
\hline No Of Nodes & $\begin{array}{c}\text { Energy In Joule } \\
\text { Proposed }\end{array}$ & $\begin{array}{c}\text { Energy In Joule } \\
\text { Heal }\end{array}$ \\
\hline 40 & 2.3 & 2.4 \\
\hline 50 & 2.5 & 3.5 \\
\hline 60 & 2.8 & 3.8 \\
\hline 70 & 2.5 & 3.8 \\
\hline 90 & 2.8 & 3.3 \\
\hline 110 & 3.1 & 3.7 \\
\hline 130 & 3.4 & 3 \\
\hline
\end{tabular}

In present system nodes are always in active mode, so energy depletion is more whereas in proposed system all nodes are in sleep mode, the node which are interested to send data are switched to active mode than send data, so proposed system requires less energy. Fig. 1 shows the graph based on values in Table 1. It appears that the energy consumption for proposed algorithm is less than existing system.

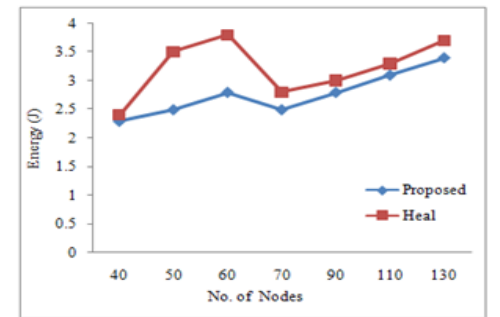

Fig. 2: Comparison of Proposed work with respect to energy consumption

\section{ACKNOWLEDGMENTS}

In this paper, fundamental issue in Wireless Sensor Network that is Hole Detection and Healing is studied. A lightweight and comprehensive protocol HEAL is presented. Which is used as holes detection in network, protocol uses distributed DHD. HEAL relocates adequate nodes within the shortest time and with lowest cost. Additionally wakeup scheduling algorithm is presented. Due to wake up scheduling energy utilization of sensor nodes is reduced and the life of network is increased. Future research can be focused on open holes located at the network boundary and also to investigate a special case of holes with obstacles.

\section{REFERENCES}

[1] Mustapha Reda Senouci, Abdelhamid Mellouk, and Khalid Assnoune, "Localized Movement-Assisted Sensor Deployment Algorithm for Hole Detection and Healing", IEEE Transactions On Parallel And DistributedSystems, Vol. 25, No. 5, pg no. 1267 $\square 1277$, May 2014

[2] C.Y. Chang, C.Y. Lin, G.J. Yu, and C.H. Kuo, “An energy Efficient Hole Healing Mechanism for Wireless Sensor Networks with Obstacles," Wireless Comm. and Mobile Computing, vol. 13, no. 4, pp. 377 $\square 392,2013$.

[3] C.Y. Chang, L.L. Hung, S.W. Chang, and Y.C. Chen, "Decentralized and Energy Balanced Algorithms for Maintaining Temporal Full Coverage in Mobile WSNs," J. Wireless Comm. and Mobile Computing , vol. 12, no.5, pp. 445-462, 2012.

[4] X. Li, G. Fletcher, A. Nayak, and I. Stojmenovic, "Randomized Carrier- Based Sensor Relocation in Wireless Sensor and Robot Networks," Proc. Ad Hoc Networks, 2012, DOI:10.1016/j.adhoc. 2012.06.007.

[5] A. Nadeem, S.K. Salil, and J. Sanjay, "A Pragmatic Approach to AreaCoverage in Hybrid Wireless Sensor Networks," Wireless Comm. And Mobile Computing, vol. 11, no. 1, pp. 23 $\square 45,2011$.

[6] B. Wang, Coverage Control in Sensor Networks. Springer, 2010.

[7] B. Wang, H.B. Lim, and M. Di, "A Survey of Movement Strategies forImproving Network Coverage in Wireless Sensor Networks," Computer Comm., vol. 32, pp. $1427 \square 1436,2009$.

[8] M. Younis and K. Akkaya, "Strategies and Techniques for Node Placement in Wireless Sensor Networks: A Survey," J. Ad Hoc Network, vol. 6, no. 4, pp. $621 \square 655$, 2008.

[9] F. Stefan and K. Christian, "Hole Detection or: How Much Geometry Hides In Connectivity" Proc. 22nd Ann. 
Symp. Computational Geometry (SCG 06), pp. 377-385, 2006.

[10] Y. Wang, J. Gao, and S.B. Mitchell, "Boundary Recognition in Sensor Networks by Topological Methods," MobiCom '06, pp. 122-133, 2006.

[11] Q. Fang, J. Gao, and L.J. Guibas, "Locating and Bypassing Holes in Sensor Networks," Mobile Networks and Applications, vol. 11, no. 2, pp. 187 $\square 200,2006$.

[12] G. Wang, G. Cao, and T.F.L. La Porta, "Movement Assisted Sensor Deployment,"IEEE Trans. Mobile Computing, vol. 5, no. 6, pp. 640 $\square 652$, June 2006.

[13] N. Ahmed, S.S. Kanhere, and S. Jha, "The Holes Problem in Wireless Sensor Networks: A Survey, SIGMOBILE Mobile Computing Comm. Rev., vol. 9, no. 2 , pp. $4 \square 18,2005$.

[14] F. Stefan, "Topological Hole Detection in Wireless Sensor Networks and its Applications," Proc. Joint Workshop on Foundations of Mobile Computing, p. $44 \square 53,2005$.
[15] S.P. Fekete, M. Kaufmann, A. Kroller, and N. Lehmann, "A New Approach for Boundary Recognition in Geometric Sensor Networks," Proc. 17th Canadian Conf. Computational Geometry, pp. 82 $\square 85,2005$.

[16] S.P. Fekete, A. Kroller, D. Pfisterer, S. Fischer, and C. Buschmann, "Neighborhood Based Topology Recognition in Sensor Networks," Proc. Int'1 Workshop on Algorithmic Aspects of Wireless Sensor Networks, pp. $123 \square 136,2004$.

[17] S. Ganeriwal, A. Kansal, and M.B. Srivastava, "Self Aware Actuation for Fault Repair in Sensor Networks," Proc. IEEE Intl Conf. Robotics and Automation (ICRA '04), pp. 5244-5249, May 2004.

[18] A. Ghosh, "Estimating Coverage Holes and Enhancing Coverage in Mixed Sensor Networks," Proc. 29th Ann. IEEE Int'l Conf. Local Computer Networks (ICLCN '04), pp. 68-76, Nov. 2004 